\title{
The Riemann Problem for a Blood Flow Model in Arteries
}

\author{
Wancheng Sheng ${ }^{1}$, Qinglong Zhang ${ }^{1, *}$ and Yuxi Zheng ${ }^{2}$ \\ ${ }^{1}$ Department of Mathematics, Shanghai University, Shanghai, 200444, P.R. China. \\ 2 Department of Mathematics, The Pennsylvania State University, University Park, \\ PA 16802, United States.
}

Received 31 August 2018; Accepted (in revised version) 31 January 2019

\begin{abstract}
In this paper, the Riemann solutions of a reduced $6 \times 6$ blood flow model in medium-sized to large vessels are constructed. The model is nonstrictly hyperbolic and non-conservative in nature, which brings two difficulties of the Riemann problem. One is the appearance of resonance while the other one is loss of uniqueness. The elementary waves include shock wave, rarefaction wave, contact discontinuity and stationary wave. The stationary wave is obtained by solving a steady equation. We construct the Riemann solutions especially when the steady equation has no solution for supersonic initial data. We also verify that the global entropy condition proposed by C.Dafermos can be used here to select the physical relevant solution. The Riemann solutions may contribute to the design of numerical schemes, which can apply to the complex blood flows.
\end{abstract}

AMS subject classifications: 35L65, 35L04, 58J45, 76N10, 35L60

Key words: Blood flow, elementary waves, Riemann problem, non-uniqueness, global entropy condition.

\section{Introduction}

A simple set of equations for the blood flow in medium-length to large arteries and veins are given by [28]

$$
\left\{\begin{array}{l}
A_{t}+(A u)_{x}=0, \\
(A u)_{t}+\left(A u^{2}\right)_{x}+\frac{A}{\rho} p_{x}=-R u,
\end{array}\right.
$$

where $A(x, t)$ is the cross section area of the vessel, $\rho, p, u$ represent the density, the pressure and the averaged velocity of the blood, respectively. We treat $\rho$ as a constant. $R$ is the

*Corresponding author. Email addresses: mathwcsheng@t.shu.edu.cn (W. Sheng), zhangqinglong@shu.edu.cn (Q. Zhang), zheng@psu.edu (Y. Zheng) 
flow resistance per unit length of the tube, assumed to be a known function here. See [27] for more details.

To complete the system, an additional condition on the pressure $p$ is provided by the tube law, which is analogous to the state equation of fluid flows. Following [23], we have the tube law

$$
p=p_{e}(x, t)+\psi\left(A ; A_{E}, K\right)
$$

where

$$
\psi\left(A ; A_{E}, K\right)=K(x)\left(\alpha^{m}-\alpha^{n}\right), \quad \alpha=\frac{A}{A_{E}} .
$$

The $p_{e}$ is the external pressure of the vessel. $K$ is the stiffness coefficient of the vessel, which represents the elastic properties of the vessel. $A_{E}$ is the cross section area at equilibrium state. $m \geq 0$ and $n \leq 0$ are two constants. For flows in arteries, $m=1 / 2, n=0$, see [22]. In this paper, we take $0<m<1, n=0$ for simplicity.

In [28], Toro and Siviglia took $p_{e}, K$ and $A_{E}$ as a function of $x$ only. Moreover, they added the following conditions to complete system (1.1)

$$
\partial_{t} p_{e}=0, \quad \partial_{t} K=0, \quad \partial_{t} A_{E}=0 .
$$

Substituting (1.3) into the second equation of (1.1), we have

$$
(A u)_{t}+\left(A u^{2}\right)_{x}+\frac{A}{\rho} \psi_{A} A_{x}+\frac{A}{\rho} \psi_{K} K_{x}+\frac{A}{\rho} \psi_{A_{E}}\left(A_{E}\right)_{x}+\frac{A}{\rho}\left(p_{e}\right)_{x}=-R u .
$$

Following Toro and Siviglia [28], we add an advection equation for a passive tracer $\phi$ representing the concentration of a chemical species. The tracer is transported passively with the fluid speed, so we have

$$
\partial_{t}(A \phi)+\partial_{x}(A u \phi)=0
$$

The advective equation is decoupled from the other equations. We note (1.6) does not add new difficulties to the Riemann problem. But for future applications, it is convenient to consider all six equations as follows

$$
\partial_{t} U+Q(U) \partial_{x} U=S(U),
$$

where $U=\left(u, A, K, A_{E}, p_{e}, \phi\right)$, and

$$
Q(U)=\left(\begin{array}{cccccc}
u & \frac{1}{\rho} \psi_{A} & \frac{1}{\rho} \psi_{K} & \frac{1}{\rho} \psi_{A_{E}} & \frac{1}{\rho} & 0 \\
A & u & 0 & 0 & 0 & 0 \\
0 & 0 & 0 & 0 & 0 & 0 \\
0 & 0 & 0 & 0 & 0 & 0 \\
0 & 0 & 0 & 0 & 0 & 0 \\
0 & 0 & 0 & 0 & 0 & u
\end{array}\right), \quad S(U)=\left(\begin{array}{c}
-\frac{R u}{A} \\
0 \\
0 \\
0 \\
0 \\
0
\end{array}\right) .
$$


System (1.7) is non-conservative, it is analogous to the flows in a variable cross section duct $[2,9,16,25]$, the two-phase flow [3], and the shallow water equations [17]. In this paper, we mainly consider the homogeneous form of (1.7), which corresponds to the non-resistance flow and is non-strictly hyperbolic because the characteristic fields may coincide with each other. For more details, we refer to $[8,10-13,16,18]$. Some numerical results can be found in $[4,5,7,14,19]$.

In [16], Lefloch and Thanh constructed the Riemann solutions of the isentropic flow in a variable cross-section duct. They divided the phase plane into subregions, in which the system is strictly hyperbolic. They also discuss the resonance and the non-uniqueness of the system. Sheng and Zhang [24] discussed the interaction of elementary waves of flows in a variable cross-section duct.

Han et al. $[10,11$ ] discussed the Riemann problem of a reduced $3 \times 3$ blood model. By using the L-M and R-M curves, which are stationary wave curves attached to two nonlinear wave curves, they constructed the Riemann solutions for the subcritical and supercritical data respectively. They also gave some numerical results showing the nonuniqueness of solutions.

The $6 \times 6$ blood flow model is analogous to the $3 \times 3$ model. Toro and Siviglia made characteristic analysis of the equations with the conditions (1.4) in [28]. They discussed the Riemann problem briefly. The resonant waves are not included in their paper. In this paper, we mainly construct the Riemann solutions of $(1.7)$ in $(u, A)$ plane. We use the classification methods in [25] to obtain the solutions according to the initial data are in different regions. The solutions lose uniqueness for some initial data. To select the physically relevant solution, we follow the global entropy condition in [6], which states that not only should the entropy increase but it should be increasing at the maximum rate. Numerical results are consistent with the criterion. In future work, we are interested in the interaction of elementary waves and constructing numerical schemes for the $6 \times 6$ blood flow model.

This paper is organized as follows. In Section 2, we make characteristic analysis of (1.7), and discuss basic properties of elementary waves, including rarefaction waves, shock waves, contact discontinuities and stationary waves. To select a unique solution of stationary discontinuity, we are not able to use the monotonicity criterion as in variable duct flows. Here we follow the conclusion obtained by [20]. In Section 3, we construct the Riemann solutions in $(u, A)$ plane. The resonance and non-uniqueness of solutions are shown in this section. In order to use the global entropy solution, we define the entropy in blood flow model. In Section 4, we design a numerical scheme for blood flow model. We also give some tests to show that the scheme preserves the mechanical energy very well.

\section{Characteristic analysis for system (1.7)}

\subsection{Preliminaries}

In this section, we consider the homogeneous form of system (1.7), namely 


$$
\partial_{t} U+Q(U) \partial_{x} U=0
$$

Considering a smooth solution of system (2.1), $Q(U)$ has six eigenvalues

$$
\lambda_{1}=u-c, \quad \lambda_{2}=u, \quad \lambda_{3}=u+c, \quad \lambda_{4}=\lambda_{5}=\lambda_{6}=0,
$$

where $c=\sqrt{\frac{A}{\rho} \psi_{A}}$. The corresponding right eigenvectors are

$$
\left\{\begin{array}{l}
\mathbf{r}_{1}=(c,-A, 0,0,0,0,)^{T}, \quad \mathbf{r}_{2}=(0,0,0,0,0,1)^{T}, \\
\mathbf{r}_{3}=(c, A, 0,0,0,0)^{T}, \quad \mathbf{r}_{4}=\left(1,-\frac{A}{u}, 0,0,-\frac{\rho\left(u^{2}-c^{2}\right)}{u}, \phi\right)^{T}, \\
\mathbf{r}_{5}=\left(0,0,1,0,-\psi_{K}, 0\right)^{T}, \quad \mathbf{r}_{6}=\left(0,0,0,1,-\psi_{A_{E}}, 0\right)^{T} .
\end{array}\right.
$$

It is easy to verify that the 2-, 4-, 5-and 6-characteristic fields are linearly degenerate, while the 1- and 3-characteristic fields are genuinely nonlinear. System (2.1) is not strictly hyperbolic because $\lambda_{1}, \lambda_{2}$ and $\lambda_{3}$ may coincide with $\lambda_{i}=0(i=4,5,6)$. More precisely, setting

$$
\Gamma_{+}: u=c, \quad \Gamma_{-}: u=-c, \quad \Gamma_{0}: u=0,
$$

we see that

$$
\lambda_{1}=\lambda_{4,5,6} \quad \text { on } \Gamma_{+}, \quad \lambda_{3}=\lambda_{4,5,6} \text { on } \Gamma_{-}, \quad \lambda_{2}=\lambda_{4,5,6} \text { on } \Gamma_{0} .
$$

In the four dimensional space $\left(u, A, K, A_{E}\right), \Gamma_{+}, \Gamma_{-}$and $\Gamma_{0}$ are three surfaces on which system (2.1) is not strictly hyperbolic. Moreover the three surfaces separate the $\left(u, A, K, A_{E}\right)$ space into four regions, denoted by $\mathrm{I}, \mathrm{II}, \mathrm{III}, \mathrm{IV}$, in which the system is strictly hyperbolic:

$$
\left\{\begin{array}{l}
\mathrm{I}=\left\{\left(u, A, K, A_{E}\right): \lambda_{4}<\lambda_{1}\right\}, \\
\mathrm{II}=\left\{\left(u, A, K, A_{E}\right): \lambda_{1}<\lambda_{4}<\lambda_{2}\right\}, \\
\mathrm{III}=\left\{\left(u, A, K, A_{E}\right): \lambda_{2}<\lambda_{4}<\lambda_{3}\right\} \\
\mathrm{IV}=\left\{\left(u, A, K, A_{E}\right): \lambda_{3}<\lambda_{4}\right\}
\end{array}\right.
$$

\subsection{Rarefaction waves}

We look for self-similar solutions depending on $\xi=\frac{x}{t}$. System (2.1) reduces to

$$
\left\{\begin{array}{l}
-\xi(A)_{\xi}+(A u)_{\xi}=0, \\
-\xi(A u)_{\xi}+\left(A u^{2}\right)_{\xi}+\frac{A}{\rho} \psi_{A} A_{\xi}+\frac{A}{\rho} \psi_{K} K_{\xi}+\frac{A}{\rho} \psi_{A_{E}}\left(A_{E}\right)_{\xi}+\frac{A}{\rho}\left(p_{e}\right)_{\xi}=0, \\
-\xi(A \phi)_{\xi}+(A \phi u)_{\xi}=0, \\
K_{\xi}=0, \\
\left(A_{E}\right)_{\xi}=0, \\
\left(p_{e}\right)_{\xi}=0 .
\end{array}\right.
$$


The Riemann invariants associated with $\lambda_{1}$ and $\lambda_{3}$ are:

$$
\begin{cases}\text { for } \lambda_{1}=u-c: & \left\{A_{E}, K, p_{e}, \phi, u+\int \frac{c(A)}{A} d A\right\}, \\ \text { for } \lambda_{3}=u+c: & \left\{A_{E}, K, p_{e}, \phi, u-\int \frac{c(A)}{A} d A\right\},\end{cases}
$$

where $c=\sqrt{\frac{A}{\rho} \psi_{A}}=\sqrt{\frac{m K}{\rho} \alpha^{m}}$, which is a function of $A, K$ and $A_{E}$. For a given left-hand state $U_{0}=\left(u_{0}, A_{0}, K_{0}, A_{E_{0}}, p_{e_{0}}, \phi_{0}\right)$, we determine the right-hand state $U=\left(u, A, K, A_{E}, p_{e}, \phi\right)$ that can be connected to $U_{0}$ by rarefaction curve as

$$
R_{1,3}\left(U, U_{0}\right):\left\{\begin{array}{l}
u \pm \frac{2 c}{m}=u_{0} \pm \frac{2 c_{0}}{m}, \quad u \geq u_{0}, \\
K=K_{0}, \quad A_{E}=A_{E_{0}}, \quad p_{e}=p_{e_{0}}, \quad \phi=\phi_{0} .
\end{array}\right.
$$

The subscripts 1 and 3 represent the backward and forward rarefaction waves respectively.

\subsection{Shock waves}

We can not define weak solution for (2.1) as usual because of the source term. However, the Rankine-Hugoniot relation associated with the variables $K, A_{E}, p_{e}$ of (2.1) is

$$
-\sigma[K]=0, \quad-\sigma\left[A_{E}\right]=0, \quad-\sigma\left[p_{e}\right]=0,
$$

where $[f]:=f_{1}-f_{0}$ is the jump of the variable $f$. We have the following conclusions:

1) $\sigma \neq 0,[K]=\left[A_{E}\right]=\left[p_{e}\right]=0$ : the variables $K, A_{E}, p_{e}$ remain unchanged across the discontinuity with non-zero speed;

2) $\sigma=0$ : the speed of discontinuity vanishes, here we assume $[f] \neq 0, f=K, A_{E}$, or $p_{e}$. We call it stationary contact discontinuity.

From 1), the Rankine-Hugoniot conditions corresponding to (1.7) is

$$
\left\{\begin{array}{l}
-\sigma[A]+[A u]=0, \\
-\sigma[A u]+\left[A u^{2}+\frac{m k}{\rho(m+1) A_{E}^{m}} A^{m+1}\right]=0, \\
-\sigma[A \phi]+[A u \phi]=0,
\end{array}\right.
$$

where $\sigma$ is the speed of the discontinuity given as

$$
\sigma_{1,3}=u_{0} \mp \frac{M}{A_{0}}, \quad M=\sqrt{\frac{m k}{\rho(m+1) A_{E}^{m}} \frac{A A_{0}\left(A^{m+1}-A_{0}^{m+1}\right)}{A-A_{0}}},
$$


and $U_{0}=\left(u_{0}, A_{0}, K_{0}, A_{E_{0}}, p_{e_{0}}, \phi_{0}\right)$ is a given left-hand state. The shock curve consisting of all right-hand state $U$ satisfying Lax shock condition is

$$
S_{1,3}\left(U, U_{0}\right):\left\{\begin{array}{l}
u=u_{0}-\sqrt{\frac{m k}{\rho(m+1) A_{E}^{m}} \frac{\left(A-A_{0}\right)\left(A^{m+1}-A_{0}^{m+1}\right)}{A A_{0}}}, \quad u<u_{0}, \\
K=K_{0}, \quad A_{E}=A_{E_{0}}, \quad p_{e}=p_{e_{0}}, \quad \phi=\phi_{0} .
\end{array}\right.
$$

Denote the 1-wave curve $W_{1}\left(U, U_{0}\right)=R_{1}\left(U, U_{0}\right) \cup S_{1}\left(U, U_{0}\right)$ and the 3-wave curve $W_{3}\left(U, U_{0}\right)=R_{3}\left(U, U_{0}\right) \cup S_{3}\left(U, U_{0}\right)$, we have the following result [10].

Lemma 2.1. For $0<m<1$, the 1-wave curve $W_{1}\left(U, U_{0}\right)$ is strictly decreasing and convex, while the 3-wave curve $W_{3}\left(U, U_{0}\right)$ is strictly increasing and concave in the $(u, A)$ plane.

Proof. We first prove the 1-wave curve $W_{1}\left(U, U_{0}\right)$ is strictly decreasing and convex. From (2.9), we have

$$
\left.\frac{\mathrm{d} u}{\mathrm{~d} A}\right|_{R_{1}\left(U, U_{0}\right)}=-\frac{c}{A}<0,\left.\quad \frac{\mathrm{d}^{2} u}{\mathrm{~d} A^{2}}\right|_{R_{1}\left(U, U_{0}\right)}=\frac{2-m}{2} \frac{c}{A^{2}}>0 .
$$

For 1-shock wave, we have

$$
\begin{aligned}
& \left.\frac{\mathrm{d} u}{\mathrm{~d} A}\right|_{S_{1}\left(U, U_{0}\right)} \\
= & -\sqrt{\frac{m K A A_{0}}{\rho(m+1) A_{E}^{m}\left(A-A_{0}\right)\left(A^{m+1}-A_{0}^{m+1}\right)}} \frac{m A^{m+1} A_{0}\left(A-A_{0}\right)+A_{0}\left(A^{m+2}-A_{0}^{m+2}\right)}{2\left(A A_{0}\right)^{2}}<0 .
\end{aligned}
$$

A straightforward but tedious calculation shows that $\left.\frac{\mathrm{d}^{2} u}{\mathrm{~d} A^{2}}\right|_{S_{1}\left(U, U_{0}\right)}>0$ for $0<m<1$. Han et al. gave a skillful proof, see [10] for details.

The proof for the 3-wave curve $W_{3}\left(U, U_{0}\right)$ is similar, we omit it.

\subsection{Contact discontinuity}

The contact discontinuity is associated with the 2-characteristic field. Across the contact discontinuity, the variables $u, A, K, A_{E}, p_{e}$ remain constant. Given the left-hand state $U_{0}$, the right-hand state $U$ that can be connected with $U_{0}$ by contact discontinuity is

$$
J\left(U, U_{0}\right): u=u_{0}, \quad A=A_{0}, \quad K=K_{0}, \quad A_{E}=A_{E_{0}}, \quad p_{e}=p_{e_{0}}, \quad \phi \neq \phi_{0} .
$$

\subsection{Stationary wave}

A stationary discontinuity is a time-independent solution. We search for the steady solution for (1.7). The stationary discontinuity solution is determined by the following 
ordinary differential equations

$$
\left\{\begin{array}{l}
(A u)_{x}=0 \\
\left(A u^{2}\right)_{x}+\frac{A}{\rho} \psi_{A} A_{x}+\frac{A}{\rho} \psi_{K} K_{x}+\frac{A}{\rho} \psi_{A_{E}}\left(A_{E}\right)_{x}+\frac{A}{\rho}\left(p_{e}\right)_{x}=0 \\
(A u \phi)_{x}=0
\end{array}\right.
$$

For smooth solutions, (2.16) is equivalent to

$$
\left\{\begin{array}{l}
(A u)_{x}=0 \\
\left(\frac{1}{2} \rho u^{2}+K\left(\left(\frac{A}{A_{E}}\right)^{m}-1\right)+p_{e}\right)_{x}=0 \\
(A u \phi)_{x}=0
\end{array}\right.
$$

Given the left hand state $U_{0}$, the right hand state $U$ connecting with $U_{0}$ by stationary discontinuity is

$$
S_{0}\left(U, U_{0}\right):\left\{\begin{array}{l}
A u=A_{0} u_{0} \\
\frac{1}{2} \rho u^{2}+K\left(\left(\frac{A}{A_{E}}\right)^{m}-1\right)+p_{e}=\frac{1}{2} \rho u_{0}^{2}+K_{0}\left(\left(\frac{A_{0}}{A_{E_{0}}}\right)^{m}-1\right)+p_{e_{0}} \\
\phi=\phi_{0} .
\end{array}\right.
$$

Substituting the first equation into the second one, we have

$$
\begin{aligned}
& \Phi\left(K, A, A_{E}, p_{e} ; A_{0}\right) \\
:= & K \frac{A^{m+2}}{A_{E}^{m}}-\left(\frac{1}{2} \rho u_{0}^{2}+K_{0}\left(\frac{A_{0}}{A_{E_{0}}}\right)^{m}+K-K_{0}+p_{e_{0}}-p_{e}\right) A^{2}+\frac{1}{2} \rho\left(A_{0} u_{0}\right)^{2}=0 .
\end{aligned}
$$

Since the variables $K, A_{E}, p_{e}$ change only across stationary discontinuity, we write $\Phi\left(K, A, A_{E}, p_{e} ; A_{0}\right)=\Phi\left(A ; A_{0}\right)$. Taking the derivative of $\Phi$ with respect to $A$, we have

$$
\frac{\mathrm{d} \Phi}{\mathrm{d} A}=(m+2) \frac{K A^{m+1}}{A_{E}^{m}}-2\left(\frac{1}{2} \rho u_{0}^{2}+K_{0}\left(\frac{A_{0}}{A_{E_{0}}}\right)^{m}+K-K_{0}+p_{e_{0}}-p_{e}\right) A .
$$

$\Phi$ reaches its minimum value at

$$
A_{\min }\left(U_{0}\right)=\left(\frac{\rho u_{0}^{2}+2 K_{0}\left(\frac{A_{0}}{A_{E_{0}}}\right)^{m}+2\left(K-K_{0}+p_{e_{0}}-p_{e}\right)}{(m+2) K}\right)^{1 / m} A_{E}
$$

We conclude that $\Phi=0$ admits a solution if and only if $\Phi\left(A_{\min }\right) \leq 0$. More precisely, we have the following lemma. 
Lemma 2.2. There exists a stationary discontinuity connecting two states $U_{0}=$ $\left(u_{0}, A_{0}, K_{0}, A_{E_{0}}, p_{e_{0}}, \phi_{0}\right)$ and $U=\left(u, A, K, A_{E}, p_{e}, \phi\right)$ if and only if $\Phi\left(A_{\min }\right) \leq 0$. Moreover, we have that

1) if $\Phi\left(A_{\min }\right)>0$, there are no stationary discontinuity;

2) if $\Phi\left(A_{\min }\right)<0$, the function $\Phi\left(A, U_{0}\right)=0$ has exactly two solutions $U_{*}\left(U_{0}\right)=$ $\left(u_{*}, A_{*}, K, A_{E}, p_{e}, \phi\right)$ and $U^{*}\left(U_{0}\right)=\left(u^{*}, A^{*}, K, A_{E}, p_{e}, \phi\right)$ with $A_{*}<A_{\min }<A^{*}$;

3) if $\Phi\left(A_{\min }\right)=0$, the two solutions $U_{*}\left(U_{0}\right)$ and $U^{*}\left(U_{0}\right)$ coincide.

We conclude that $u_{*}>\mathcal{c}_{*}=\sqrt{\frac{m K}{\rho}\left(\frac{A_{*}}{A_{E}}\right)^{m}}, u^{*}<c^{*}=\sqrt{\frac{m K}{\rho}\left(\frac{A^{*}}{A_{E}}\right)^{m}}$. i.e., the two solutions $U_{*}$ and $U^{*}$ are in the supercritical area $(u>c)$ and subcritical area $(u<c)$ respectively. In fact, from (2.21), we have

$$
A_{*}<A_{\min }\left(U_{0}\right)<A^{*},
$$

which yields $u_{*}^{2}>c_{*}^{2}$ and $u^{* 2}<c^{* 2}$.

Remark 2.1. The condition $\Phi\left(A_{\min }\right) \leq 0$ may not hold in some cases. For example, when the difference between $p_{e_{0}}$ and $p_{e}$ is very large, or the variable $K$ is large enough compared to $K_{0}, p_{e_{0}}$ and $A_{E}$. The flow will across a shock wave to reduce the speed and make $\Phi\left(A_{\min }\right) \leq 0$ solvable under these cases. The explicit solution will be discussed in Section 3.

As in $[3,10,25]$, the Riemann problem for (2.1) may admit up to a one-parameter family of solutions. To select a unique solution, their principle can be simplified as: The flow is subcritical (supercritical) at one side of the stationary discontinuity iff it is also subcritical (supercritical) at the other side of it.

Siviglia and Toffolon [20] considered the steady flow in blood vessels with discontinuous mechanical properties of a collapsible tube. They concluded that in arteries, the transition of subcritical state to supercritical state is not possible across the steady waves. They also proposed a principle to choose the stationary solutions. Basically, they defined the momentum flux

$$
g(A):=\rho \frac{H^{2}}{A}+A\left(p_{e}+\psi\right)-\int_{A_{0}}^{A} \psi \mathrm{d} A,
$$

where $H=A u$ remains constant across the stationary discontinuity. The physically admissible solution is selected where $g(A)$ has the minimum value. Generally, a smooth transition between subcritical solution to supercritical solution only happens under critical conditions, i.e., the state on one side of the stationary discontinuity is critical: $u_{0}=c_{0}$. Following their results, we have the following lemma.

Lemma 2.3. For the $6 \times 6$ system (2.1), across the stationary discontinuity, the states on the two sides remain in the closure of only one domain in $(u, A)$ plane. 


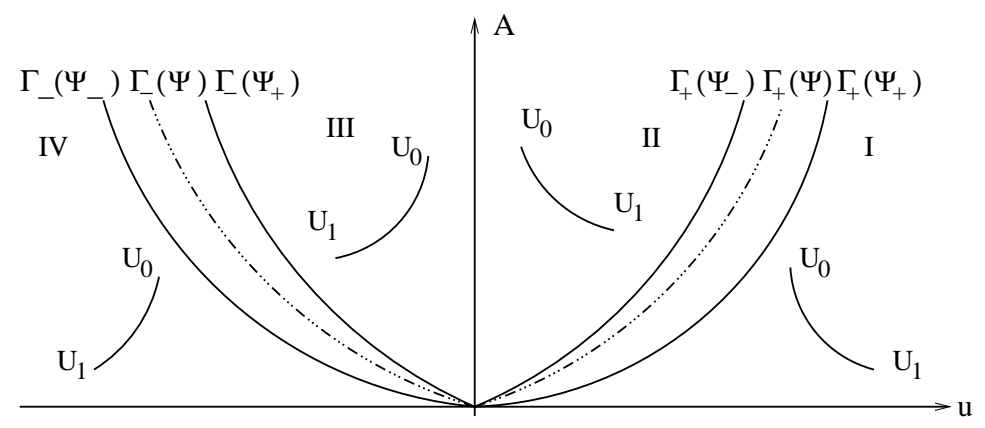

Figure 1: The stationary curve remains in one domain in $(u, A)$ plane.

Under the assumption of Lemma 2.3, we call the stationary discontinuity as stationary wave, denoted by $S_{0}\left(U, U_{0}\right)$ from the left hand state $U_{0}$ to the right hand state $U$. We note that the sonic curve $\Gamma_{ \pm}(\Psi)$ is a function of $K$ and $A_{E}$. The stationary wave curve will change continuously on one side of $\Gamma_{ \pm}(\Psi)$ from Lemma 2.3. See Fig. 1.

For stationary wave $S_{0}\left(U, U_{0}\right)$, Lemma 2.3 tells us that when $U_{0}$ is subcritical, we choose $U_{0}^{*}$ as the solution. When $U_{0}$ is supercritical, $U_{0 *}$ is chosen as the solution. For the critical case $u_{0}=c_{0}$, there are two solutions, one is subcritical, the other is supercritical. The selection of the two solutions will be discussed in Section 3.

Remark 2.2. For blood flows in veins, Lemma 2.3 may not hold, which causes multiple solutions [21] and further research is needed.

\subsection{Shock wave coincides with stationary wave}

In order to construct the Riemann solution, we have to know when the shock speed equals to zero. When $u_{0}<c_{0}$, from (2.11), we have

$$
\begin{aligned}
\sigma_{1}<c_{0}-\frac{M}{A_{0}} & =\sqrt{\frac{m K}{\rho}\left(\frac{A_{0}}{A_{E}}\right)^{m}}-\sqrt{\frac{m K}{\rho(m+1) A_{E}^{m}} \frac{A\left(A^{m+1}-A_{0}^{m+1}\right)}{A_{0}\left(A-A_{0}\right)}} \\
& =\frac{1}{N} \frac{m K}{\rho A_{0} A_{E}^{m}}\left(A_{0}^{m+1}-A A_{\theta}^{m}\right)<0, \quad A_{0}<A_{\theta}<A,
\end{aligned}
$$

where

$$
N=\sqrt{\frac{m K}{\rho}\left(\frac{A_{0}}{A_{E}}\right)^{m}}+\sqrt{\frac{m K}{\rho(m+1) A_{E}^{m}} \frac{A\left(A^{m+1}-A_{0}^{m+1}\right)}{A_{0}\left(A-A_{0}\right)}} .
$$

From (2.11), let $\sigma_{1}=0$, by using the relation $u_{0}=\frac{A u}{A_{0}}, A>A_{0}$, we have

$$
u^{2}=\frac{A_{0} m K}{A \rho(m+1) A_{E}^{m}} \frac{A^{m+1}-A_{0}^{m+1}}{A-A_{0}}=\frac{A_{0}}{A} \frac{m K}{\rho}\left(\frac{A_{\theta}}{A_{E}}\right)^{m}<c^{2}, \quad A_{0}<A_{\theta}<A .
$$


Combining (2.24) and (2.25), we have $\sigma_{1}<0$ when $U_{0} \in \Pi \cup I I \cup I V$. When $U_{0} \in \mathrm{I}$, there exists a point $\widetilde{U}_{0} \in \Pi$ such that $\sigma_{1}\left(\widetilde{U}_{0}, U_{0}\right)=0$. For 3 -shock wave $S_{3}\left(U, U_{0}\right)$, there exists a point $\widetilde{U}_{0} \in \mathrm{III}$ such that $\sigma_{3}=0$ if $U_{0} \in \mathrm{IV}$. Therefore we have the following lemma.

Lemma 2.4. 1) The 1-shock speed $\sigma_{1}\left(U, U_{0}\right)$ may change its sign along the shock curve $S_{1}\left(U, U_{0}\right)$. More precisely,

i) If $U_{0} \in \Pi \cup \mathrm{III} \cup \mathrm{IV}$, then $\sigma_{1}\left(U, U_{0}\right)$ remains negative

$$
\sigma_{1}\left(U, U_{0}\right)<0 .
$$

ii) If $U_{0} \in \mathrm{I}$, then there exists a point $U=\widetilde{U}_{0} \in \Pi$ on the shock curve $S_{1}\left(U, U_{0}\right)$ such that

$$
\left\{\begin{array}{l}
\sigma_{1}\left(\widetilde{U}_{0}, U_{0}\right)=0, \\
\sigma_{1}\left(U, U_{0}\right)>0, A \in\left(A_{0}, \widetilde{A}_{0}\right), \\
\sigma_{1}\left(U, U_{0}\right)<0, \quad A \in\left(\widetilde{A}_{0},+\infty\right) .
\end{array}\right.
$$

2) The 3-shock speed $\sigma_{3}\left(U, U_{0}\right)$ may change its sign along the shock curve $S_{3}\left(U, U_{0}\right)$, i.e., i) If $U_{0} \in I \cup I I \cup I I I$, then $\sigma_{3}\left(U, U_{0}\right)$ remains positive

$$
\sigma_{3}\left(U, U_{0}\right)>0 .
$$

ii) If $U_{0} \in I V$, then there exists a point $U=\widetilde{U}_{0} \in \mathrm{II}$ on the shock curve $S_{3}\left(U, U_{0}\right)$ such that

$$
\left\{\begin{array}{l}
\sigma_{3}\left(\widetilde{U}_{0}, U_{0}\right)=0, \\
\sigma_{3}\left(U, U_{0}\right)<0, \quad A \in\left(A_{0}, \widetilde{A}_{0}\right), \\
\sigma_{3}\left(U, U_{0}\right)>0, \quad A \in\left(\widetilde{A}_{0},+\infty\right) .
\end{array}\right.
$$

Now we are ready to construct the Riemann solution of blood flow model (1.1). It is well known that the Riemann solution is not unique and there are up to three solutions for some initial data $[2,11,16]$. How to select a unique physically relevant solution is still an open problem. Here we follow Dafermos [6] and Andrianov and Warnecke [2], where a global entropy condition is proposed which states that not only should the entropy increase but it should be increasing at the maximum rate. First we use the entropy defined as [6]:

$$
S=\frac{1}{2} u^{2}+\frac{K}{\rho(m+1)} \sqrt{\frac{A}{A_{e}}}
$$

in system (1.1). We note that (2.28) is derived from the conservation laws. But it can be applied to nonconservative system as well [1]. Then we give the global entropy condition in blood flow model.

Global entropy condition. In blood flow model (1.1), the physically relevant solution of system (1.1) is chosen such that the entropy defined in (2.28) not only increase but also increase at the maximum rate.

Under the global entropy condition, we can pick out the unique physical solution. Some numerical results are shown in Section 4. 


\section{The Riemann problem of system (2.1)}

In this section, we consider the Riemann problem of system (2.1) with two pieces of constant initial data

$$
\left.U(x, t)\right|_{t=0}=\left.\left(u, A, K, A_{E}, p_{e}, \phi\right)\right|_{t=0}= \begin{cases}\left(u_{-}, A_{-}, K_{-}, A_{E-}, p_{e-}, \phi_{-}\right), & x<0 \\ \left(u_{+}, A_{+}, K_{+}, A_{E+}, p_{e+}, \phi_{+}\right), & x>0 .\end{cases}
$$

We will establish the global existence of the Riemann problem for (2.1) with (3.1). Under the self-similar transformation $\xi=x / t$, the Riemann problem reduces to a boundary value problem of ordinary differential equations (2.7) with

$$
\left(u, A, K, A_{E}, p_{e}, \phi\right)( \pm \infty)=\left(u_{ \pm}, A_{ \pm}, K_{ \pm},\left(A_{E}\right)_{ \pm},\left(p_{e}\right)_{ \pm}, \phi_{ \pm}\right) .
$$

We will use the following notations:

(i) $W_{1}\left(U_{j}, U_{i}\right) \oplus W_{3}\left(U_{k}, U_{j}\right)$ indicates that there is a backward wave $W_{1}$ from the lefthand state $U_{i}$ to the right-hand state $U_{j}$, followed by a forward wave $W_{3}$ from the left-hand state $U_{j}$ to the right-hand state $U_{k}$.

(ii) $\bar{U}$ denotes the right-hand state from the left-hand state $U=\left(u, A, K, A_{E}, p_{e}, \phi\right)$ by contact discontinuity $J(\bar{U}, U)$ associated to $\lambda_{2}=u$. In $(u, A)$ plane, $U$ and $\bar{U}$ denote the same point.

(iii) Denote a vector $\Omega=\left(K, A_{E}, p_{e}\right)$. $\Omega$ only changes across stationary wave which satisfies (2.18).

The Riemann problem of a reduced $3 \times 3$ blood model is discussed by Han et al. [10, $11]$, in which they classify the initial data into subcritical and supercritical in phase plane respectively. Here we give a different approach. Since the stationary wave $S_{0}$ always has zero speed, we classify the Riemann solutions based on the $(x, t)$ plane. Noticing that for blood flow in veins, we can also use this method to construct the Riemann solution. The only difference is that in veins, the transition through critical condition is likely to occur due to an abrupt change of the mechanical properties of the wall vessel [21]. However, a straightforward and similar calculation shows that we still have Lemma 2.4 holds in veins, which means the sonic curve $\Gamma_{ \pm}(\Psi)$ can still be used to classify the solution. More specifically, the Riemann solutions in $(x, t)$ plane have the following structures:

1) $W_{1}, J$ and $W_{3}$ are on one side of $S_{0}$;

2) $W_{1}, J$ and $W_{3}$ are on both sides of $S_{0}$;

3) Either $W_{1}$ or $W_{3}$ coincides with $S_{0}$;

4) Two stationary waves are included in the solutions. 
Case 1. $W_{1}, J$ and $W_{3}$ are on one side of $S_{0}$, which includes two cases: $S_{0} \oplus W_{1} \oplus J \oplus W_{3}$ and $W_{1} \oplus J \oplus W_{3} \oplus S_{0}$. We discuss them separately.

Subcase 1.1. $U_{-} \in \mathrm{I}, U_{+} \in \mathrm{I}$ and some part of II. $U_{-}$first jumps to $U_{-*} \in \mathrm{I}$ by stationary wave $S_{0}$ with $\Omega$ shifting from $\Omega_{-}$to $\Omega_{+}$, see Fig. 2 . By Lemma 2.4 , there exists a point $\widetilde{U}_{-*} \in \Pi$ on $S_{1}\left(U, U_{-*}\right)$ such that the shock speed vanishes, i.e., $\sigma_{1}\left(\widetilde{U}_{-*}, U_{-*}\right)=0$. Denote $\left\{U_{2}\right\}=W_{3}\left(U_{+}, U\right) \cap W_{1}\left(U, U_{-*}\right)$. If $\widetilde{u}_{-*} \leq u_{2}$, the Riemann solution is

$$
S_{0}\left(U_{-*}, U_{-}\right) \oplus W_{1}\left(U_{2}, U_{-*}\right) \oplus J\left(\bar{U}_{2}, U_{2}\right) \oplus W_{3}\left(U_{+}, \bar{U}_{2}\right) .
$$
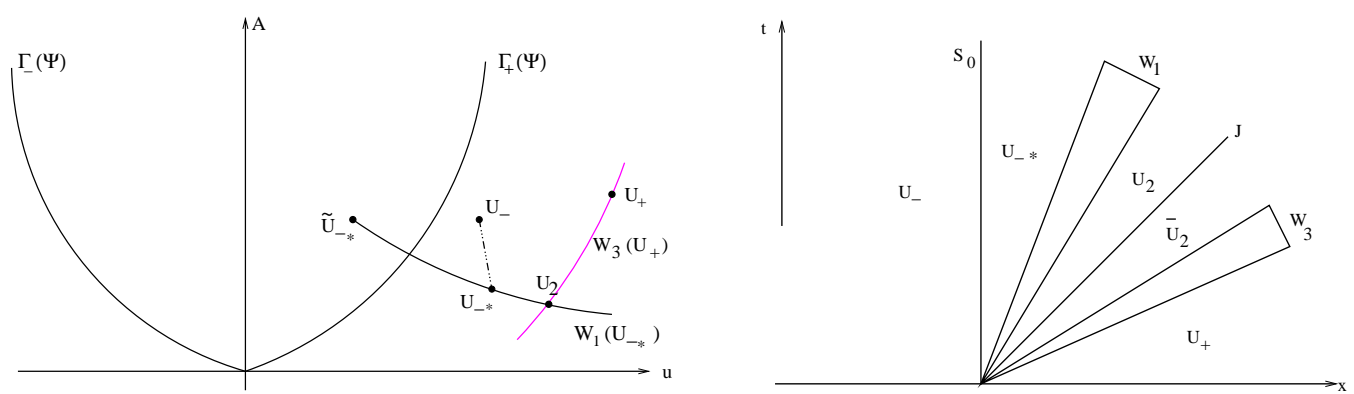

Figure 2: Subcase 1.1 and wave configuration.

Subcase 1.2. $U_{+} \in I V, U_{-} \in I V$ and some part of III. See Fig. 3. Denote $U_{+*} \in I V$ jumped by $U_{+}$from the right side with stationary wave. There exists $\widetilde{U}_{+*} \in$ II satisfying $\sigma_{3}\left(\widetilde{U}_{+*}, U_{+*}\right)=0$ by Lemma 2.4. Denote $\left\{U_{3}\right\}=W_{1}\left(U, U_{-}\right) \cap W_{3}\left(U_{+*}, U\right)$. If $u_{3} \leq \tilde{u}_{+*}$, The solution is

$$
W_{1}\left(U_{3}, U_{-}\right) \oplus J\left(\bar{U}_{3}, U_{3}\right) \oplus W_{3}\left(U_{+*}, \bar{U}_{3}\right) \oplus S_{0}\left(U_{+}, U_{+*}\right) .
$$
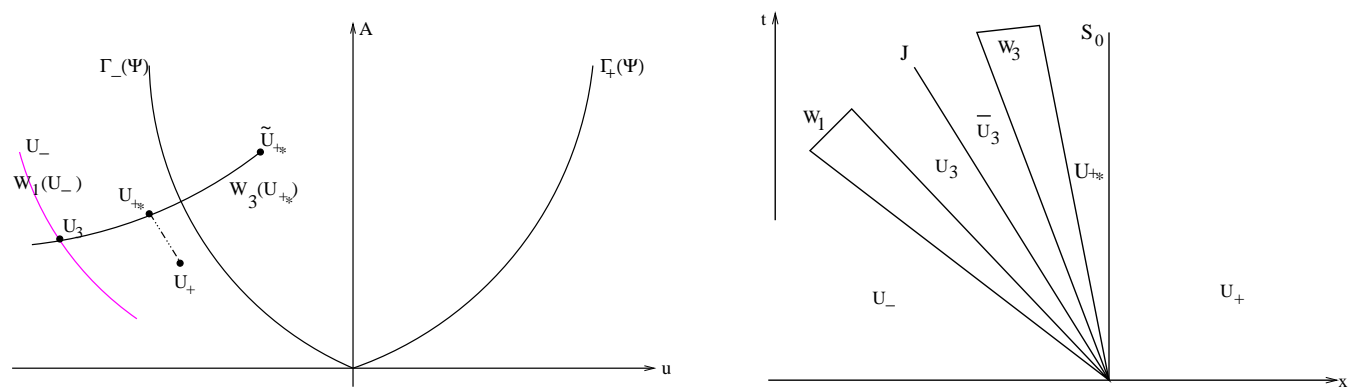

Figure 3: Subcase 1.2 and wave configuration.

Remark 3.1. If $u_{+}-\frac{2}{m} c_{+} \geq u_{-*}+\frac{2}{m} c_{-*}$ in Subcase 1.1 or $u_{-}+\frac{2}{m} c_{-} \leq u_{+*}-\frac{2}{m} c_{+*}$ in Subcase 1.2 , there exists a vacuum. As shown in $[10,22]$, the collapse of vessel in arteries may not appear under normal physiological conditions. Thus the vacuum solution only has academic meaning. 
Case 2. $W_{1}, J$ and $W_{3}$ are on both sides of $S_{0}$. The solution can be $W_{1} \oplus S_{0} \oplus J \oplus W_{3}$ or $W_{1} \oplus J \oplus S_{0} \oplus W_{3}$. We discuss as follows:

Subcase 2.1. $U_{-} \in$ I. Denote $\widetilde{U}_{-} \in I$ II on $S_{1}\left(U, U_{-}\right)$such that the shock speed vanishes, i.e., $\sigma_{1}\left(\widetilde{U}_{-}, U_{-}\right)=0$. $\widetilde{U}_{-}$jumps to $\widetilde{U}_{-}^{*}$ by stationary wave using subcritical solution. Denote $U_{0} \in S_{1}\left(U, \widetilde{U}_{-}\right), u_{0}<\tilde{u}_{-}$, the state $U_{1}$ obtained by stationary jump from $U_{0}$ forms a curve $S_{0}\left(U_{1}, U_{0}\right)$. See Fig. 4 (left). Denote $\left\{U_{4}^{*}\right\}=W_{3}\left(U_{+}, U\right) \cap S_{0}\left(U_{1}, U_{0}\right), U_{4}^{*}$ is jumped by stationary wave from $U_{4} \in S_{1}\left(U, U_{-}\right)$if $u_{4}^{*}>0$ (or from $\bar{U}_{4} \in J\left(U, U_{4}\right)$ if $u_{4}^{*}<0$ ). This construction holds for $U_{+} \in I \cup I I I$ or $U_{+} \in I V, \sigma_{3}\left(U_{4}^{*}, U_{+}\right)>0$. See Fig. 5. We have

1) If $u_{4}^{*} \geq 0$, the solution is

$$
S_{1}\left(U_{4}, U_{-}\right) \oplus S_{0}\left(U_{4}^{*}, U_{4}\right) \oplus J\left(\overline{U_{4}^{*}}, U_{4}^{*}\right) \oplus W_{3}\left(U_{+}, \overline{U_{4}^{*}}\right) .
$$

2) If $u_{4}^{*}<0$, the solution is

$$
S_{1}\left(U_{4}, U_{-}\right) \oplus J\left(\bar{U}_{4}, U_{4}\right) \oplus S_{0}\left(U_{4}^{*}, \bar{U}_{4}\right) \oplus W_{3}\left(U_{+}, U_{4}^{*}\right)
$$
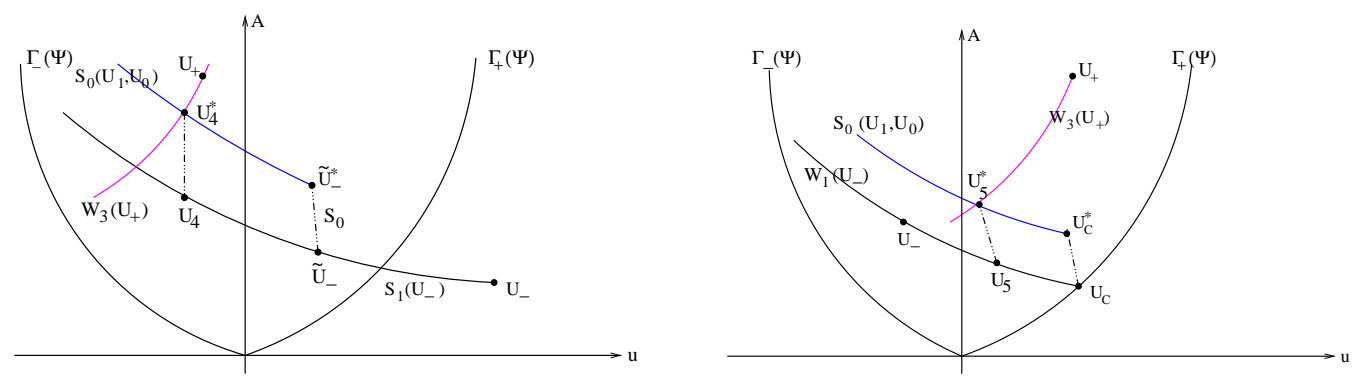

Figure 4: Subcase 2.1 (left) and Subcase 2.2 (right).
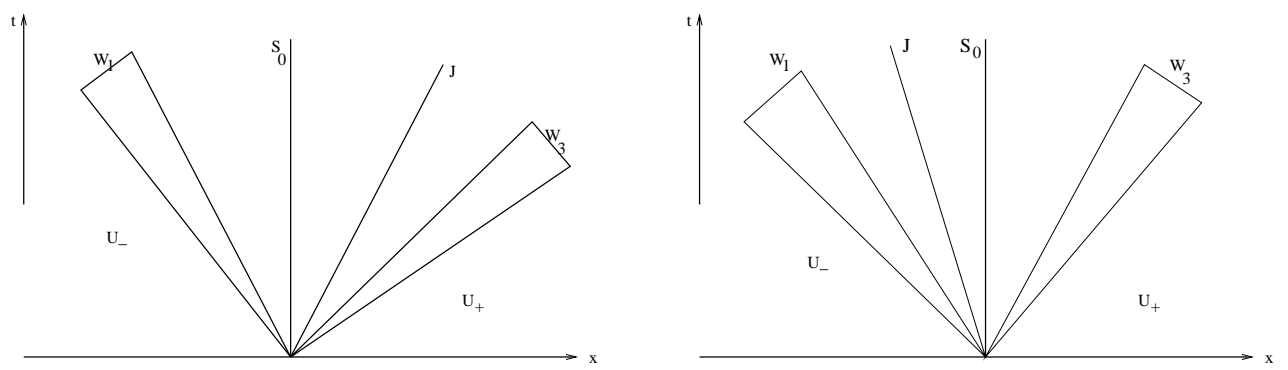

Figure 5: Wave configuration for Subcase 2.1 and Subcase 2.2.

Subcase 2.2. $U_{-} \in \Pi \cup$ III. Denote $\left\{U_{C}\right\}=W_{1}\left(U, U_{-}\right) \cap \Gamma_{+}(\Psi)$. $U_{C}$ jumps to $U_{C}^{*} \in I$ by stationary wave. Denote $U_{0} \in W_{1}\left(U, U_{-}\right)\left(u_{0} \leq u_{c}\right)$, the state $U_{1}$ obtained by stationary wave from $U_{0}$ forms a curve $S_{0}\left(U_{1}, U_{0}\right)$. See Fig. 4 (right). Denote $\left\{U_{5}^{*}\right\}=W_{3}\left(U, U_{+}\right) \cap$ 
$S_{0}\left(U_{1}, U_{0}\right), U_{5}^{*}$ is jumped from $U_{5} \in S_{1}\left(U, U_{-}\right)$if $u_{5}^{*}>0$ (or from $\bar{U}_{5}$ if $u_{5}^{*}<0$ ). Similarly, if $U_{+} \in \Pi \cup \mathrm{III}$ or $U_{+} \in \mathrm{IV}, \sigma_{3}\left(U_{5}^{*}, U_{+}\right)>0$, we have

1) If $u_{5}^{*} \geq 0$, the solution is

$$
W_{1}\left(U_{5}, U_{-}\right) \oplus S_{0}\left(U_{5}^{*}, U_{5}\right) \oplus J\left(\overline{U_{5}^{*}}, U_{5}^{*}\right) \oplus W_{3}\left(U_{+}, \overline{U_{5}^{*}}\right) .
$$

2) If $u_{5}^{*}<0$, the solution is

$$
W_{1}\left(U_{5}, U_{-}\right) \oplus J\left(\bar{U}_{5}, U_{5}\right) \oplus S_{0}\left(U_{5}^{*}, \bar{U}_{5}\right) \oplus W_{3}\left(U_{+}, U_{5}^{*}\right) .
$$

Case 3. Either $W_{1}$ or $W_{3}$ coincides with $S_{0}$. The solution can be $W_{1} \oplus S_{0} \oplus W_{1} \oplus J \oplus W_{3}$ or $W_{1} \oplus J \oplus W_{3} \oplus S_{0} \oplus W_{3}$. We discuss as follows:
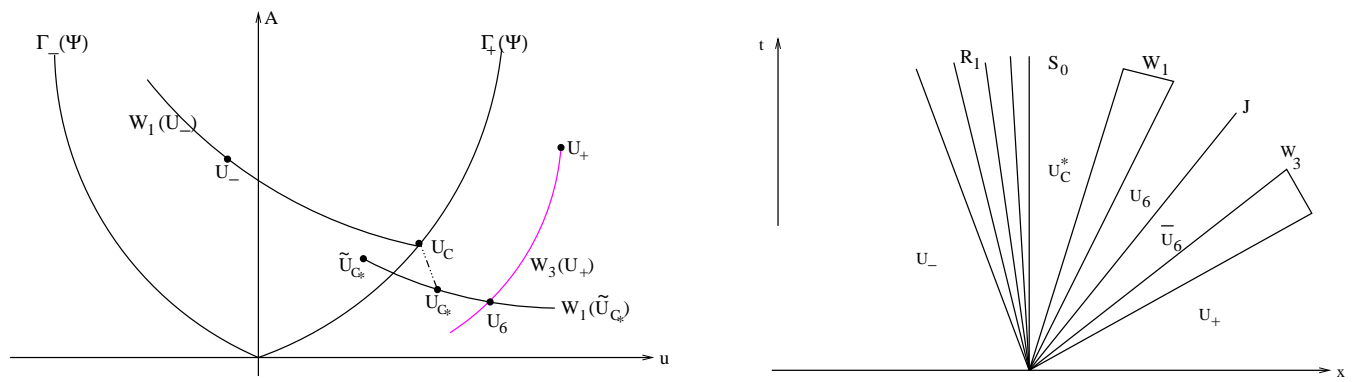

Figure 6: Subcase 3.1 and wave configuration.

Subcase 3.1. $U_{-} \in \Pi \cup \Pi \amalg \cup I V, U_{+} \in I$ and some part of II. $U_{-}$passes through a 1-wave first. Denote $\left\{U_{C}\right\}=W_{1}\left(U, U_{-}\right) \cap \Gamma_{+}, U_{C}$ jumps to $U_{C *} \in I$ by stationary wave. There exists a point $\widetilde{U}_{C *} \in S_{1}\left(U, U_{C *}\right)$ satisfying $\sigma_{1}\left(\widetilde{U}_{C *}, U_{C *}\right)=0$. See Fig. 6. Denote $\left\{U_{6}\right\}=$ $W_{1}\left(U, U_{C *}\right) \cap W_{3}\left(U, U_{+}\right)$. If $u_{6} \geq \tilde{u}_{c *}$, the solution is

$$
R_{1}\left(U_{C}, U_{-}\right) \oplus S_{0}\left(U_{C *}, U_{C}\right) \oplus W_{1}\left(U_{6}, U_{C *}\right) \oplus J\left(\bar{U}_{6}, U_{6}\right) \oplus W_{3}\left(U_{+}, \bar{U}_{6}\right) .
$$
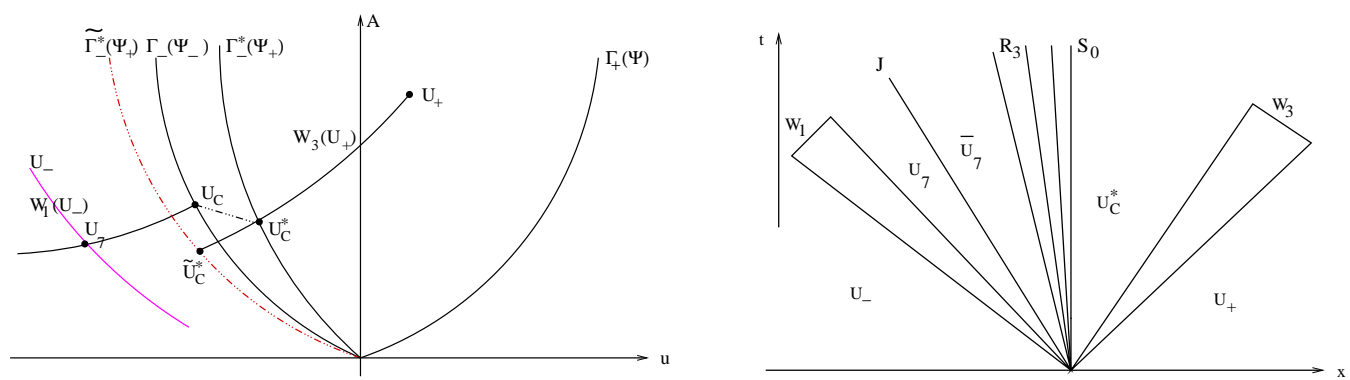

Figure 7: Subcase 3.2 and wave configuration.

Subcase 3.2. $U_{-} \in I V, U_{+} \in I \cup I \cup I I I$ and some part of IV. First we denote two curves in $(u, A)$ plane: $\Gamma_{-}^{*}\left(\Psi_{+}\right)$and $\widetilde{\Gamma}_{-}^{*}\left(\Psi_{+}\right) . \Gamma_{-}^{*}\left(\Psi_{+}\right)$is formed with states jumping from $\Gamma_{-}\left(\Psi_{-}\right)$ 
by stationary wave using subcritical solutions. For any $U_{0} \in \Gamma_{-}^{*}\left(\Psi_{+}\right) \in \mathrm{III}$, there exists a point $U_{1} \in \mathrm{III}$ on $S_{3}\left(U, U_{0}\right)$ satisfying $\sigma_{3}\left(U_{1}, U_{0}\right)=0$, the state $U_{1}$ forms the curve $\widetilde{\Gamma_{-}^{*}}\left(\Psi_{+}\right)$. Denote $\left\{U_{C}^{*}\right\}=W_{3}\left(U_{+}, U\right) \cap \Gamma_{-}^{*}\left(\Psi_{+}\right), U_{C *}$ is jumped by $U_{C} \in \Gamma_{-}\left(\Psi_{-}\right)$. See Fig. 7 . Denote $\left\{U_{7}\right\}=W_{1}\left(U, U_{-}\right) \cap R_{3}\left(U_{C}, U_{7}\right)$. If $U_{+} \in \mathrm{I} \cup \mathrm{II} \cup \mathrm{III}$ or $U_{+} \in \mathrm{IV}, \sigma_{3}\left(U_{C^{*}}^{*} U_{+}\right)>0$, then the solution is

$$
W_{1}\left(U_{7}, U_{-}\right) \oplus J\left(\bar{U}_{7}, U_{7}\right) \oplus R_{3}\left(U_{C}, \bar{U}_{7}\right) \oplus S_{0}\left(U_{C}^{*}, U_{C}\right) \oplus W_{3}\left(U_{+}, U_{C}^{*}\right) .
$$

Subcase 3.3. $U_{-} \in \mathrm{I}, U_{+} \in \mathrm{I}$. We consider an interesting coinciding case. When the initial data (3.1) is given such that the function $\Phi$ defined in (2.19) has no solution. We construct the solution as follows: $U_{-}$first jumps to $U_{C}^{*} \in \Pi$ by a shock wave, then $U_{C}^{*} \in \Pi$ jumps to $U_{C}$ on $\Gamma_{+}(\Psi)$ by a stationary wave, followed by a backward rarefaction wave connecting $U_{C}$ and $U_{2}, U_{2}$ jumps to $\bar{U}_{2}$ by a contact discontinuity, finally $\bar{U}_{2}$ and $U_{+}$are connected by a 3 -wave $W_{3}\left(U, U_{+}\right)$. See Fig. 8 . The solution is

$$
S_{1}\left(U_{C}^{*}, U_{-}\right) \oplus S_{0}\left(U_{C}, U_{C}^{*}\right) \oplus R_{1}\left(U_{2}, U_{C}\right) \oplus J\left(\bar{U}_{2}, U_{2}\right) \oplus W_{3}\left(U_{+}, \bar{U}_{2}\right) .
$$
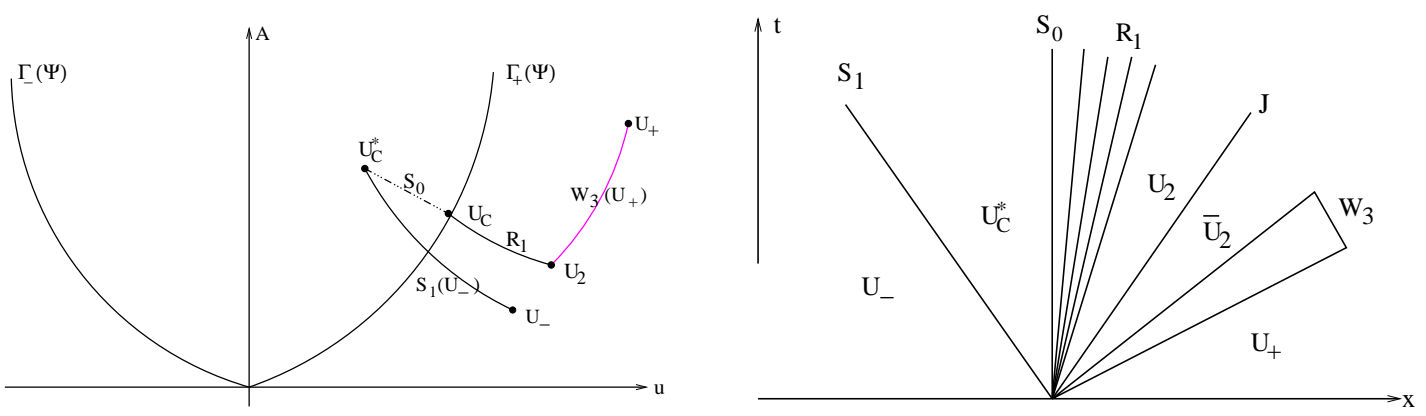

Figure 8: Subcase 3.3 and wave configuration.

Remark 3.2. We extend this construction to a more general case when $\Phi$ has no solution. We claim that the Riemann solution involves a shock wave to decrease the speed of blood flow in this case. In fact, for arbitrary initial data $U_{0}$, we can prove that there exists a state $U_{1} \in S_{1}\left(U, U_{0}\right)$ such that $\Phi\left(U, U_{1}\right)$ admits a solution. An extremely case is that the flow is at rest across the shock wave, thus (2.19) has a solution.

Case 4. In this case we consider a solution contains three waves with the same zero speed, i.e., two stationary waves and a standing 1-shock wave. This case holds for $U_{-} \in \mathrm{I}$, $U_{+} \in I \cup I I \cup I I I \cup I V$. See Fig. 9. First we have two points $\widetilde{U}_{-}^{*}, \widetilde{U}_{-*} \in \mathbb{I I}$, see Subcase 1.1 and Subcase 2.1 for the definition. From (2.10) and (2.18), we can easily derive $\widetilde{A}_{-}^{*} \tilde{u}_{-}^{*}=$ $\widetilde{A}_{-*} \tilde{u}_{-*}=A_{-} u_{-}$, this is exactly the conservation of mass. Thus $\widetilde{U}_{-}^{*}, \widetilde{U}_{-*}$ are on the curve $A u=A_{-} u_{-}$. We have two cases: $\tilde{u}_{-}^{*}<\tilde{u}_{-*}$ and $\tilde{u}_{-}^{*}>\tilde{u}_{-*}$.

Subcase 4.1. $\tilde{u}_{-}^{*}<\tilde{u}_{-*}$, see Fig. 9 (left). From $U_{-}$, the solution jumps to a state $U_{1}\left(\Omega_{0}\right)$ by stationary wave with an intermediate value $\Omega_{0} \in\left[\Omega_{-}, \Omega_{+}\right]$. Then $U_{1}\left(\Omega_{0}\right)$ connects with 

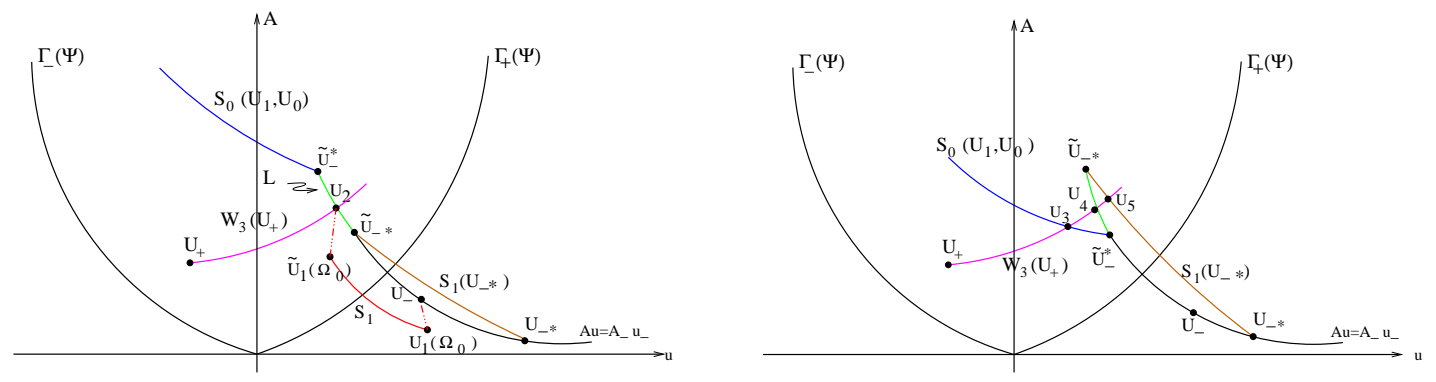

Figure 9: Subcase 4.1 and wave configuration.

$\widetilde{U}_{1}\left(\Omega_{0}\right) \in$ II by a standing 1 -shock wave $S_{1}$. Next, $\widetilde{U}_{1}\left(\Omega_{0}\right)$ jumps to $U_{1}\left(\Omega_{+}\right)$by another stationary wave with $\Omega$ shifting from $\Omega_{0}$ to $\Omega_{+}$.

As shown in Thanh [25], set

$$
L:=\left\{U(\Omega) \mid \Omega \in\left[\Omega_{-}, \Omega_{+}\right]\right\} .
$$

If $W_{3}\left(U_{+}, U\right) \cap L=\left\{U_{2}\right\}$, then the solution contains three discontinuities having the same speed zero, see Fig. 9 (left). The solution is

$$
S_{0}\left(U_{1}\left(\Omega_{0}\right), U_{-}\right) \oplus S_{1}\left(\widetilde{U}_{1}\left(\Omega_{0}\right), U_{1}\left(\Omega_{0}\right)\right) \oplus S_{0}\left(U_{2}, \widetilde{U}_{1}\left(\Omega_{0}\right)\right) \oplus J\left(\bar{U}_{2}, U_{2}\right) \oplus W_{3}\left(U_{+}, \bar{U}_{2}\right) .
$$

We note that the solution is unique in this case.

Subcase 4.2. $\tilde{u}_{-}^{*}>\tilde{u}_{-*}$, see Fig. 9 (right). We see that $W_{3}\left(U_{+}, U\right)$ may intersect with $S_{1}\left(U, U_{-*}\right), S_{0}\left(U_{1}, U_{0}\right)$ and $L$ at the same time. The solution lose uniqueness and has up to three solutions. Namely, Subcase 1.1, Subcase 2.1 and Subcase 4.1. We use the global entropy condition in Section 2 to pick out the physical relevant solution. Moreover, we will show that the numerical results are consistent with the global entropy condition in Section 4 . Which is to say, the physically relevant solution is the one that maximizes $S$ in (2.28).

\section{Numerical simulations}

In this section, we will develop a numerical scheme for the model (2.1), which is built in [15] for compressible flows in a variable cross-section duct. It has been proved that the scheme captures exactly equilibrium states. Given a uniform time step $\Delta t$ and an equal mesh size $\Delta x$. Set $x_{j}=j \Delta x, j \in \mathbf{Z}, t^{n}=n \Delta t, n \in \mathbf{N}$. Let $U_{j}^{n}$ be the approximation of the values $U\left(x_{j}, t^{n}\right)$ of the exact solution. Our well-balanced scheme for (2.1) is defined by

$$
U_{j}^{n+1}=U_{j}^{n}-\frac{\Delta t}{\Delta x}\left(g\left(U_{j}^{n}, U_{j+1,-}^{n}\right)-g\left(U_{j-1,+}^{n}, U_{j}^{n}\right)\right),
$$


where $U_{j+1,-}^{n}$ and $U_{j-1,+}^{n}$ are given shortly below. $g(U, V)$ can be any standard numerical flux for blood flow model. Here we take the Lax-Friedrichs flux:

$$
\left\{\begin{array}{l}
g(U, V)=\frac{1}{2}(f(U)+f(V))-\frac{1}{2 \lambda}(V-U), \\
U:=(A, A u, A \phi), \quad f(U):=\left(A u, A u^{2}+\frac{m k}{\rho(m+1) A_{E}^{m}} A^{m+1}, A u \phi\right) .
\end{array}\right.
$$

In the scheme (4.1), the states

$$
U_{j+1,-}^{n}=(A, A u, A \phi)_{j+1,-}^{n}, \quad U_{j-1,+}^{n}=(A, A u, A \phi)_{j-1,+}^{n}
$$

are defined from the mechanical energy equations (2.17). We compute $A_{j+1,-}^{n}$ and $u_{j+1,-}^{n}$ from the equations

$$
\left\{\begin{array}{l}
A_{j+1}^{n} u_{j+1}^{n}=A_{j+1,-}^{n} u_{j+1,-}^{n} \\
\frac{1}{2} \rho\left(u_{j+1}^{n}\right)^{2}+K_{j+1}^{n}\left(\left(\frac{A_{j+1}^{n}}{\left(A_{E}\right)_{j+1}^{n}}\right)^{m}-1\right)+\left(p_{e}\right)_{j+1}^{n} \\
\quad=\frac{1}{2} \rho\left(u_{j+1,-}^{n}\right)^{2}+K_{j}^{n}\left(\left(\frac{A_{j+1,-}^{n}}{\left(A_{E}\right)_{j}^{n}}\right)^{m}-1\right)+\left(p_{e}\right)_{j}^{n},
\end{array}\right.
$$

and compute $A_{j-1,+}^{n}$ and $u_{j-1,+}^{n}$ from the equations

$$
\left\{\begin{array}{l}
A_{j-1}^{n} u_{j-1}^{n}=A_{j-1,+}^{n} u_{j-1,+^{\prime}}^{n} \\
\frac{1}{2} \rho\left(u_{j-1}^{n}\right)^{2}+K_{j-1}^{n}\left(\left(\frac{A_{j-1}^{n}}{\left(A_{E}\right)_{j-1}^{n}}\right)^{m}-1\right)+\left(p_{e}\right)_{j-1}^{n} \\
\quad=\frac{1}{2} \rho\left(u_{j-1,+}^{n}\right)^{2}+K_{j}^{n}\left(\left(\frac{A_{j-1,+}^{n}}{\left(A_{E}\right)_{j}^{n}}\right)^{m}-1\right)+\left(p_{e}\right)_{j}^{n} .
\end{array}\right.
$$

Remember we have the steady solutions

$$
\left\{\begin{array}{l}
A_{j+1}^{n} u_{j+1}^{n}=A_{j}^{n} u_{j \prime}^{n} \\
\frac{1}{2} \rho\left(u_{j+1}^{n}\right)^{2}+K_{j+1}^{n}\left(\left(\frac{A_{j+1}^{n}}{\left(A_{E}\right)_{j+1}^{n}}\right)^{m}-1\right)+\left(p_{e}\right)_{j+1}^{n} \\
\quad=\frac{1}{2} \rho\left(u_{j}^{n}\right)^{2}+K_{j}^{n}\left(\left(\frac{A_{j}^{n}}{\left(A_{E}\right)_{j}^{n}}\right)^{m}-1\right)+\left(p_{e}\right)_{j}^{n} .
\end{array}\right.
$$

Therefore, the unique solutions for (4.4) and (4.5) will be

$$
\begin{cases}A_{j+1,-}^{n}=A_{j,}^{n}, & u_{j+1,-}^{n}=u_{j}^{n}, \\ A_{j-1,+}^{n}=A_{j,}^{n}, & u_{j-1,+}^{n}=u_{j}^{n},\end{cases}
$$


which indicates (see (4.1))

$$
U_{j}^{n+1}=U_{j}^{n}
$$

This means we exactly recover the steady solutions.

In a domain where $K, A_{E}, p_{e}$ are constant, it is not difficult to verify that

$$
U_{j+1,-}^{n}=U_{j+1}^{n}, \quad U_{j-1,+}^{n}=U_{j-1}^{n} .
$$

This implies that (4.1) reduced to the standard Lax-Friedrichs scheme where $K, A_{E}, p_{e}$ are constant.

In our tests, we use the computing correctors $d_{j}^{n}>0$ introduced in [26] at the discrete level. $d_{j}^{n}$ is defined by

$$
d_{j}^{n}=\Delta x \cdot \max \left|\lambda_{i}\left(U_{j}^{n}\right)\right|\left(\left|A_{j+1}^{n}-A_{j}^{n}\right|+\left|u_{j+1}^{n}-u_{j}^{n}\right|\right), \quad i=1,2, \cdots, 6,
$$

which will enhance scheme (4.1) especially in resonant regime.

Now we give some numerical simulations of the Riemann solution involving stationary wave using the scheme (4.1). The results are consistent with that in Section 3. For the other case, we note that it is the same with Euler equation and will not discuss here. Take the blood density $\rho=1050 \mathrm{~kg} / \mathrm{m}^{3}, m=0.5$. The CFL constant is 0.5 . We use 2000 mesh points. The solution will be computed for $x \in[0,20]$, the stationary discontinuity is located at $x=10$. Here $U=\left(u, A, K, A_{E}, p_{e}, \phi\right)$.

Case 1. The initial data is

$$
U_{-}=(2.5,0.0003,2000,0.002,2.0,2.0), \quad U_{+}=(2.1685,0.00034586,1000,0.001,1.0,0.5) .
$$

We have $U_{-} \in \mathrm{I}, U_{+} \in \mathrm{I}$. The result is obtained at time $t=1.25 \mathrm{~s}$. The solution contains a stationary wave and a contact discontinuity. See Fig. 10 (left). We see that the schemes captures exactly the steady solution. It is the same as the Riemann solution in the Subcase 1.1.

Case 2. The initial data is

$$
U_{-}=(2.5,0.0003,2000,0.002,2.0,2.0), \quad U_{+}=(2.25,0.0008,1000,0.001,1.0,0.5) .
$$

We have $U_{-} \in \mathrm{I}, U_{+} \in \mathrm{I}$. The result is obtained at time $t=1.5 \mathrm{~s}$. The solution begins with a stationary wave, followed by a backward shock wave, followed by a contact discontinuity, then followed by a forward rarefaction wave. See Fig. 10 (right). It is the same as the Riemann solution in the Subcase 1.1.

Case 3. The initial data is

$$
U_{-}=(0.75,0.00015,1000,0.000015,3.0,3.0), \quad U_{+}=(3.5,0.0002,2000,0.0001,1.0,1.5) .
$$



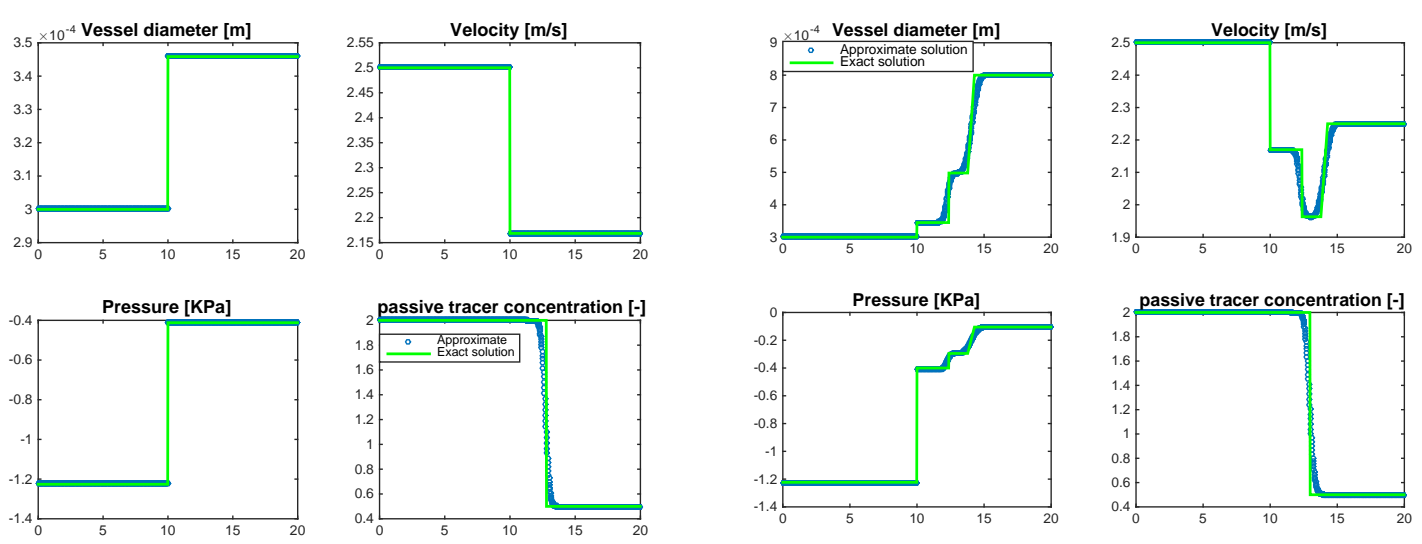

Figure 10: Case 1 (left). $S_{0} \oplus J$. Case 2 (right). $S_{0} \oplus S_{1} \oplus J \oplus R_{3}$.
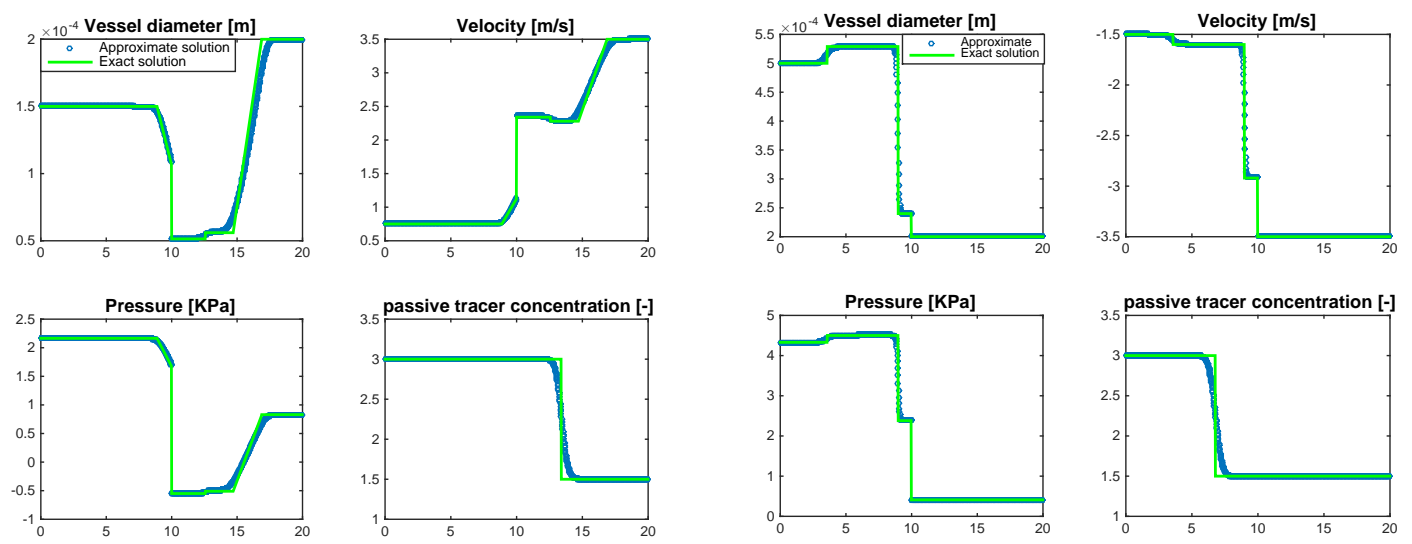

Figure 11: Case 3 (left). $R_{1} \oplus S_{0} \oplus S_{1} \oplus J \oplus R_{3}$. Case 4 (right). $S_{1} \oplus J \oplus S_{3} \oplus S_{0}$.

We have $U_{-} \in \Pi, U_{+} \in \mathrm{I}$. The result is obtained at time $t=1.5 \mathrm{~s}$. The solution begins with a backward rarefaction wave, followed by a stationary wave, followed by a backward shock wave, followed by a contact discontinuity, then followed by a forward rarefaction wave. See Fig. 11 (left). It is the same as the Riemann solution in Case 3.

Case 4. The initial data is

$$
U_{-}=\left(-1.5,0.0005,2000,5 \times 10^{-5}, 3.0,3.0\right), \quad U_{+}=\left(-3.5,0.0002,1000,10^{-4}, 1.0,1.5\right) .
$$

We have $U_{-} \in \mathrm{III}, U_{+} \in \mathrm{IV}$. The result is obtained at time $t=2.0$ s. The solution begins with a backward shock wave, followed by a contact discontinuity, followed by a forward shock wave, then followed by a stationary wave. See Fig. 11 (right). It is the same as the Riemann solution in the Subcase 1.2. 
Case 5. The initial data is

$$
U_{-}=\left(0.1,0.0002,1200,10^{-5}, 1.0,3.0\right), \quad U_{+}=\left(0.2,0.0006,1000,10^{-5}, 5.0,2.0\right) .
$$

We have $U_{-} \in \mathrm{I}, U_{+} \in \mathrm{II}$. Fig. 12 is the result obtained at time $t=2.5 \mathrm{~s}$. The solution begins with a backward shock wave, followed by a stationary wave, followed by a contact discontinuity, then followed by a forward rarefaction wave. See Fig. 12. It is the same as the Riemann solution in Case 2.
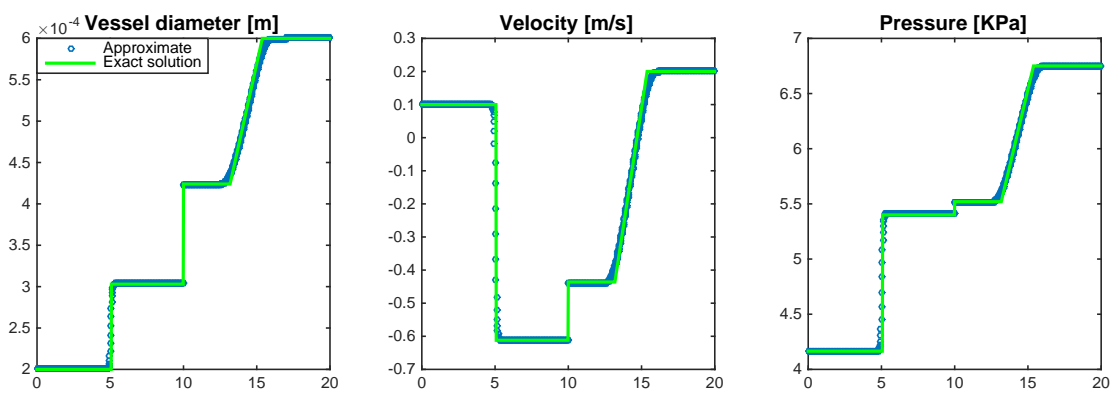

Figure 12: Case 5. $S_{1} \oplus S_{0} \oplus J \oplus R_{3}$.

Case 6. The initial data is

$$
\begin{aligned}
& U_{-}=\left(-0.01323213,10^{-5}, 0.290682,10^{-2}, 3.0,3.0\right), \\
& U_{+}=\left(0.050242,1.243 \times 10^{-6}, 0.24708,10^{-2}, 3.0,3.0\right) .
\end{aligned}
$$

We have $U_{-} \in \mathrm{IV}, U_{+} \in \mathrm{I}$. The result is obtained at time $t=80.0 \mathrm{~s}$. The solution begins with a backward rarefaction wave, followed by a stationary wave, followed by a contact discontinuity, then followed by a forward rarefaction wave. We see that the vessel collapse in this case. See Fig. 13. It is the same as the Riemann solution in Case 2.
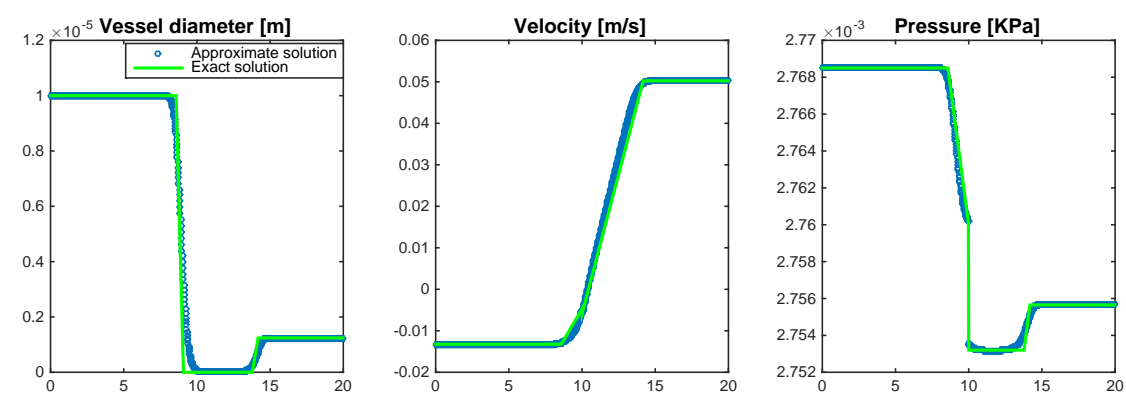

Figure 13: Case 6. $R_{1} \oplus$ Vacuum $\oplus S_{0} \oplus J \oplus R_{3}$.

Case 7. The initial data is

$$
U_{-}=\left(2.0,0.00025,100,10^{-4}, 3.0,0.0\right), \quad U_{+}=\left(0.48125,0.000925,1000,10^{-4}, 3.0,100.0\right) \text {. }
$$



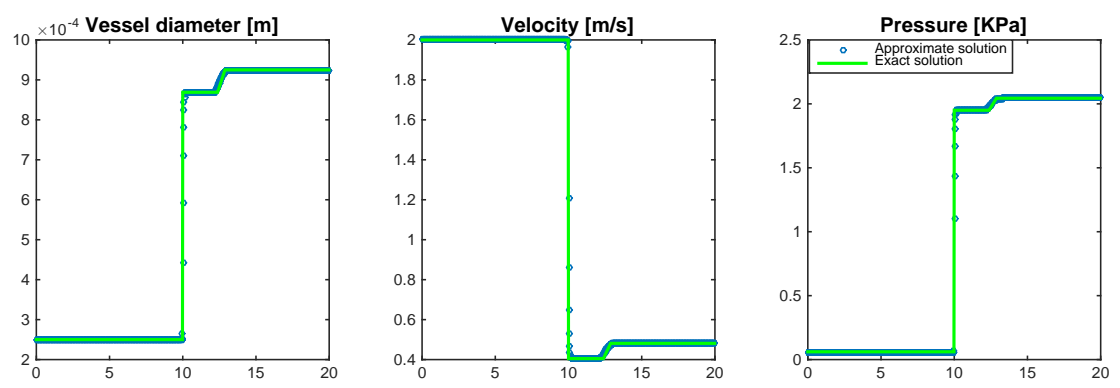

Figure 14: Case 7. $S_{0} \oplus S_{1} \oplus S_{0} \oplus J \oplus R_{3}$.

We have $U_{-} \in \mathrm{I}, U_{+} \in \mathrm{II}$. The result is obtained at time $t=1.5 \mathrm{~s}$. The solution contains three discontinuities with the same zero speed, followed by a contact discontinuity, then followed by a forward rarefaction wave. See Fig. 14. It is the same as the Riemann solution in the Subcase 4.1.

The next two examples are taken from Han et al. [11]. The initial data are chosen such that the solutions are not unique as in the Subcase 4.2. The multiple solutions are computed in Han et al. [11] for each example. We will see that our new scheme captures the solutions exactly. We also use the examples to verify the physically relevant solution is the one that maximizes the entropy defined in (2.28).

Case 8. The initial data is

$$
\begin{aligned}
& U_{-}=\left(6.655409,10^{-6}, 58136.483963,10^{-4}, 3.0,3.0\right), \\
& U_{+}=\left(0.0,5.038 \times 10^{-6}, 56392.389444,10^{-4}, 3.0,3.0\right) .
\end{aligned}
$$

We have $U_{-} \in \mathrm{I}, U_{+} \in \mathrm{I}$. Fig. 15 is the result obtained at time $t=2.0 \mathrm{~s}$. The solution begins with a stationary wave, followed by a backward shock wave, followed by a contact discontinuity, then followed by a forward shock wave. See Fig. 15. We see that the new scheme captures the entropy solution very well.
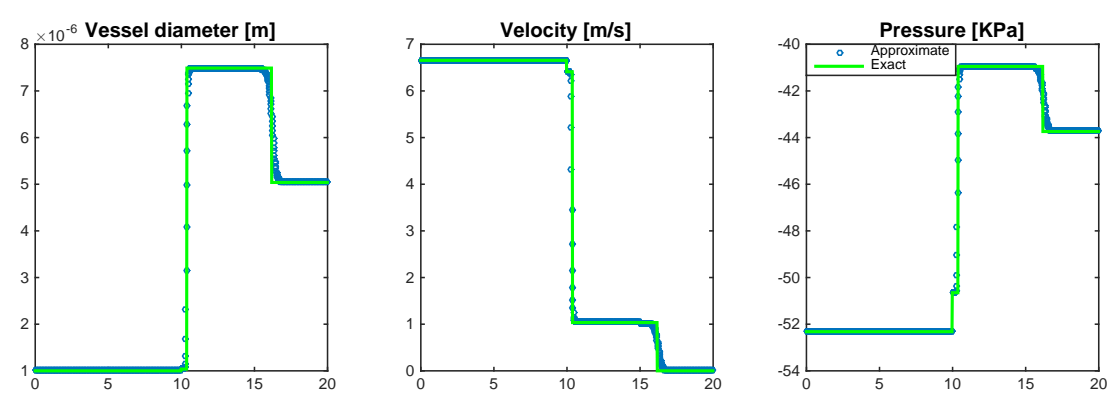

Figure 15: Case 8. $S_{0} \oplus S_{1} \oplus J \oplus S_{3}$. 
Case 9. The initial data is

$$
\begin{aligned}
& U_{-}=\left(0.023530423,10^{-6}, 0.919219,10^{-4}, 3.0,1.0\right), \\
& U_{+}=\left(0.022542384,10^{-5}, 0.781336,10^{-4}, 3.0,1.0\right) .
\end{aligned}
$$

We have $U_{-} \in \mathrm{I}, U_{+} \in \mathrm{I}$. Fig. 16 is the result obtained at time $t=150$ s. The solution begins with a stationary wave, followed by a backward shock wave, followed by a contact discontinuity, then followed by a forward rarefaction wave. See Fig. 16.
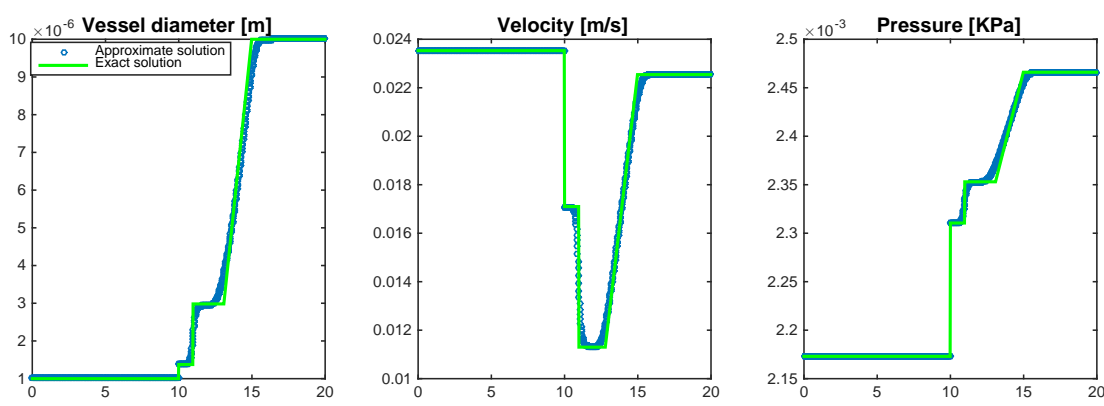

Figure 16: Case 9. $S_{0} \oplus S_{1} \oplus J \oplus R_{3}$.

In summary, we construct the Riemann solutions of a $6 \times 6$ blood flow model using phase plane analysis methods. When steady equation (2.19) has no solution for supersonic initial data, we also construct the solution in this case for the first time. The solution lose uniqueness and there exist up to three solutions for the same initial data. To follow the global entropy condition, we propose the entropy in blood flow model. We develop a numerical scheme which is showed to preserve the mechanical energy very well.

\section{Acknowledgments}

This work was supported by NSFC 11371240 and 11771274 . Zhang was also supported by the State Scholarship Fund from China Scholarship Council (201706890042). The authors gratefully acknowledge Professor Jiequan Li for his enthusiastic help.

\section{References}

[1] N. Andrianov, Analytical and Numerical Investigation of Two Phase Flows, PhD Thesis (Otto-von-Guericke University, Magdeburg 2003).

[2] N. Andrianov and G. Warnecke, On the solution to the Riemann problem for the compressible duct flow, SIAM J. Appl. Math., 64(2004), 878-901.

[3] N. Andrianov and G. Warnecke, The Riemann problem for Baer-Nunziato model of two phase flows, J. Comput. Phys., 195(2004), 434-464. 
[4] W. Boscheri and R. Loubère, High Order Accurate Direct Arbitrary-Lagrangian-Eulerian ADER-MOOD Finite Volume Scheme for Non-Conservative Hyperbolic Systems with Stiff Source Terms, Commun. Comput. Phys., 21(2017), 271-312.

[5] V. Caleffi, A. Valiani and G. Li, A comparison between bottom-discontinuity numerical treatments in the DG framework, Appl. Math. Model., 40(2016), 7516-7531.

[6] C. Dafermos, The entropy rate admissibility criterion for solutions of hyperbolic conservation laws, J. Diff. Eqs., 14(1973), 202-212.

[7] S. Gao and T. G. Liu, A Solution Structure-Based Adaptive Approximate (SSAA) Riemann Solver for the Elastic-Perfectly Plastic Solid, Commun. Comput. Phys., 25(2019), 781-811.

[8] H. M. Glaz, T. P. Liu, The asymptotic analysis of wave interactions and numerical calculations of transonic nozzle flow, Adv. in Appl. Math. 5(1984), 111-146.

[9] E. Han and M. Hantke and G. Warnecke, Exact Riemann solutions in ducts with discontinuous cross-section, J. Hyp. Diff. Equations, 9(2012), 403-449.

[10] E. Han, G. Warnecke, E.F. Toro, A. Siviglia, On Riemann solutions to weakly hyperbolic systems: part 1. Modelling subcritical flows in arteries, technical report NI15003NPA, Isaac Newton Institute for Mathematical Sciences, University of Cambridge, UK, 2015a.

[11] E. Han, G. Warnecke, E.F. Toro, A. Siviglia, On Riemann Solutions to Weakly Hyperbolic Systems: Part 2. Modelling Supercritical Flows in Arteries, Technical Report NI15004NPA, Isaac Newton Institute for Mathematical Sciences, University of Cambridge, UK, 2015b.

[12] E. Isaacson and B. Temple, Nonlinear resonance in systems of conservation laws, SIAM J. Appl. Math., 52(1992), 1260-1278.

[13] E. Isaacson and B. Temple, Convergence of the $2 \times 2$ Godunov method for a general resonant nonlinear balance law, SIAM J. Appl. Math., 55(1995), 625-640.

[14] S. Jin, A steady-state capturing method for hyperbolic systems with geometrical source terms, Math. Model. Numer. Anal., 35 (2001), 631-645.

[15] D. Kröner and M. D. Thanh. Numerical solutions to compressible flows in a nozzle with variable cross-section. SIAM J. Numer. Anal., 43(2005), 796-824.

[16] P. G. LeFloch and M. D. Thanh, The Riemann problem for fluid flows in a nozzle with discontinuous cross-section, Commun. Math. Sci., 1(2003), 763-797.

[17] P. G. LeFloch and M. D. Thanh, A Godunov-type method for the shallow water equations with discontinuous topography in the resonant regime, J. Comput. Phys., 230(2011), 76317660 .

[18] T.P. Liu, Nonlinear stability and instability of transonic flows through a nozzle, Comm. Math. Phys., 83(1982), 243-260.

[19] A. Navas-Montilla and J. Murillo, Asymptotically and exactly energy balanced augmented flux-ADER schemes with application to hyperbolic conservation laws with geometric source terms, J. Comput. Phys., 317(2016), 108-147.

[20] A. Siviglia and M. Toffolon, Steady analysis of transcritical flows in collapsible tubes with discontinuous mechanical properties: implications for arteries and veins, J. Fluid Mech., 736(2013), 195-215.

[21] A. Siviglia and M. Toffolon, Multiple states for flow through a collapsible tube with discontinuities, J. Fluid Mech., 761(2014), 105-122.

[22] C. Spiller, E. F. Toro, M. E. Vázquez-Cendeón, C. Contarino. On the exact solution of the Riemann problem for blood flow in human veins, including collapse, Applied Mathematics and Computation, 303(2017), 178-189.

[23] A. H. Shapiro, Steady flow in collapsible tubes. Trans. ASME: J. Biomech. Engng 99, 1977, 126-147. 
[24] W. C. Sheng and Q. L. Zhang, Interaction of the elementary waves of isentropic flow in a variable cross-section duct, Commun. Math. Sci. 16(6)(2018), 1659-1684.

[25] M. D. Thanh, The Riemann problem for a nonisentropic fluid in a nozzle with discontinuous cross-sectional area, SIAM Journal on Applied Mathematics, 69(2009), 1501-1519.

[26] M. D. Thanh and D. Kröner, Numerical treatment of nonconservative terms in resonant regime for fluid flows in a nozzle with variable cross-section, Comput. Fluids, 66(2012), 130139

[27] E. F. Toro and A. Siviglia. Simplified blood flow model with discontinuous vessel properties: analysis and exact solutions. In: Modelling of Physiological Flows (D. Ambrosi, A. Quarteroni, G. Rozza, Eds), Springer-Verlag, 2011.

[28] E. F. Toro and A Siviglia, Flow in collapsible tubes with discontinuous mechanical properties: mathematical model and exact solutions, Commun. Comput. Phys., 13(2013), 361-385. 